\title{
The prevalence of low energy availability in active females in Ireland
}

\author{
D.M. Logue ${ }^{1,2}$, S.M. Madigan ${ }^{2}$, E. Delahunt ${ }^{1}$, M. Heinen ${ }^{1}$, S.J. Mc Donnell ${ }^{2}$ and C.A. Corish ${ }^{1}$ \\ ${ }^{1}$ School of Public Health, Physiotherapy and Sports Science, University College Dublin, Belfield, Dublin, Republic of \\ Ireland and \\ ${ }^{2}$ Sport Ireland Institute, Abbottstown, Dublin, Republic of Ireland
}

Energy availability (EA) has been defined as the amount of ingested energy remaining to maintain normal bodily function and physiological processes such as thermoregulation, immunity, growth and locomotion after the energy required for exercise/training has been removed. In a state of low energy availability (LEA), whereby there is insufficient energy available for normal physiological functioning, adaptations may occur which can potentially contribute to unfavourable health and performance outcomes ${ }^{(1,2)}$. As different methods have been used to determine the extent of this problem, the prevalence of LEA has yet to be definitely established in active females.

The aim of this cross-sectional study was to determine the prevalence of LEA risk among active females in Ireland using an online questionnaire validated for use in females: the 'Low Energy Availability in Females Questionnaire' (LEAF-Q) ${ }^{(3)}$. Twenty nine additional questions collected information on demographics, dietary habits, as well as injury and illness history. A chi-squared test was used to compare categorical frequencies.

Nine hundred active females living in Ireland completed the online questionnaire between November 2016 and February 2017. The data were entered and cleaned in Excel [Microsoft Excel 2013 (KB3115162)] and transported into SPSS version 12.0.2 (IBM Corporation) for analysis. Fifty two participants were excluded as they did not meet current Irish recommendations for physical activity. A total of $39.6 \%(n, 336)$ of respondents were categorised as "at risk" of LEA. The table outlines the estimated prevalence of LEA risk in the different groups, which constituted the sample of respondents.

\begin{tabular}{lrrrr}
\hline & & & \multicolumn{2}{c}{ LEA Risk } \\
\cline { 5 - 5 } & $(n)$ & $(\%)$ & $(n)$ & $(\%)$ \\
\hline Sporting Level & & & & \\
International & 164 & $19 \cdot 3$ & 74 & 22 \\
Provincial & 48 & $5 \cdot 7$ & 22 & $6 \cdot 5$ \\
Inter-county & 109 & $12 \cdot 9$ & 51 & $15 \cdot 2$ \\
Recreational & 241 & $28 \cdot 4$ & 78 & $23 \cdot 2$ \\
Participate competitively & 286 & $33 \cdot 7$ & 111 & 33 \\
\hline
\end{tabular}

More athletes $(P \leqslant 0 \cdot 01)$ competing in sport $(76.8 \%)$ were at risk of LEA compared with those who were recreationally active $(23 \cdot 2 \%)$. Those at risk of LEA missed more than eight days of training in the previous year due to injury and illness compared to those not at risk (injury: $73.6 \%$ vs. $42.1 \%, P \leqslant 0.001$; illness: $49.3 \%$ vs. $37.3 \%, P \leqslant 0.01$ ). Participants "at risk" reported more daily/weekly bloating and cramps unrelated to menstruation compared to those who were "not at risk" (bloating/gaseous: $33.9 \% \mathrm{vs} .12 .5 \%, P \leqslant 0.001$; cramps: $21.7 \%$ vs. $3.9 \%, P \leqslant 0.001$ ). The high prevalence for the risk of LEA highlights the need to improve awareness of this condition, as well as the development of appropriate EA management strategies to optimise the health and performance of athletes.

1. Mountjoy M, Sundgot-Borgen J, Burke L et al. (2014) Br J Sports Med. 7, 491-497.

2. Nattiv A, Loucks AB, Manore MM et al. (2007) Med Sci Sports Exerc. 10, 1867-1882.

3. Melin A, Tornberg A, Skouby S et al. (2014) Br J Sports Med. 7, 540-545. 